\title{
Bipolaron in the $t-J$ model coupled to longitudinal and transverse quantum lattice vibrations
}

\author{
L. Vidmar, ${ }^{1}$ J. Bonča, ${ }^{2,1}$ S. Maekawa, ${ }^{3,4}$ and T. Tohyama ${ }^{5}$ \\ ${ }^{1} \mathrm{~J}$. Stefan Institute, 1000 Ljubljana, Slovenia \\ ${ }^{2}$ Faculty of Mathematics and Physics, University of Ljubljana, 1000 Ljubljana, Slovenia \\ ${ }^{3}$ Institute for Materials Research, Tohoku University, Sendai 980-857r, Japan \\ ${ }^{4}$ CREST, Japan Science and Technology Agency (JST), Tokyo 102-0075, Japan \\ ${ }^{5}$ Yukawa Institute for Theoretical Physics, Kyoto University, Kyoto 606-8502, Japan
}

(Dated: November 14, 2018)

\begin{abstract}
We explore the influence of two different polarizations of quantum oxygen vibrations on the spacial symmetry of the bound magnetic bipolaron in the context of the $t-J$ model by using exact diagonalization within a limited functional space. Linear as well as quadratic electron phonon coupling to transverse polarization stabilize $d$-wave symmetry. The existence of a magnetic background is essential for the formation of a $d$-wave bipolaron state. With increasing linear electron phonon coupling to longitudinal polarization the symmetry of a $d$-wave bipolaron state changes to a $p$-wave. Bipolaron develops a large anisotropic effective mass.

PACS numbers: 71.27.+a,71.38.Mx, 71.38.-k,74.20.Rp
\end{abstract}

Soon after the discovery of high- $T_{c}$ superconductivity the quest for the pairing mechanism focused on magnetic fluctuations due to a broadly accepted conjecture that phonon mechanism alone is not strong enough to produce high transition temperatures as observed in high$T_{c}$ compounds. Recently, a growing evidence is emerging in favor of the significance of lattice degrees of freedom in high- $T_{c}$ compounds [1, 2, 3]. The interplay between strong correlations and lattice degrees of freedom [4] seems to be responsible for many unusual properties of cuprates in the low doping regime, such as kinks [1], stripes [5], and waterfall [6, 7] structures.

A long-standning objection against phonon-based mechanism for high- $T_{c}$ superconductivity is based on a widely accepted notion that coupling to phonon degrees of freedom is predominantly consistent with $s$-wave pairing, not characteristic for cuprates. Despite recent discovery that weak EP coupling to acoustic phonons in the presence of large on-site Coulomb interaction leads to $d$-wave pairing [8], the role of short-wavelength oxygen oscillations on the symmetry of the paired state remains a challenging open problem.

Investigation of correlated models coupled to phonons were based on exact diagonalization (ED) calculations on small lattice systems [9, 10, 11, 12], slave-boson approaches 13, 14, 15], dynamical mean-field calculations 16, 17, 18], coherent states Lanczos method [19], and quantum Monte Carlo methods (QMC) [12, 20]. In Ref. 9] authors present a detailed study of the influence of EP coupling to a single phonon mode on formation of inhomogeneous charge structures in the $t-J$ model. They show that half-breathing mode stabilizes a stripe phase. In contrast, using slave-boson approach authors of Ref. [13] suggest, that half-breathing mode enhances $d$ - wave pairing. They furthermore underline the importance of off-diagonal EP coupling modulating the hopping and the spin-exchange terms. In contrast, authors of Ref. 21] find that diagonal EP terms exceed off-diagonal ones by nearly two orders of magnitude.

ED calculations of the $t-J$ model show that the $d$-wave symmetry of a bipolaron [22, 23, 24, 25, 26] is not robust against addition of longer range hopping terms [27] while recent QMC calculations of the Hubbard model yield $T_{c}$ far below those of cuprates [28]. There seems to be a need to uncover additional mechanism that would help stabilize the $d$-wave symmetry of a bound bipolaron state. In this Letter we show that EP coupling to a transverse polarization (TP) of oxygen vibration provides an important mechanism that stabilizes the $d$-wave symmetry of a bound hole pair with a small effective mass.

We solve a system of two holes in the $t-J$ model defined on an infinite two-dimensional lattice by extending the method for a single hole based on exact diagonalization within a limited functional space [7, 29]. We introduce diagonal EP coupling to either TP of oxygen (O) vibration relevant for the description of buckling modes or longitudinal polarization (LP) of $\mathrm{O}$ vibration relevant for description of bond-streching modes. We investigate the following Hamiltonian

$$
\begin{aligned}
H & =-t \sum_{\langle\mathbf{i}, \mathbf{j}\rangle, s}\left(\tilde{c}_{\mathbf{i}, s}^{\dagger} \tilde{c}_{\mathbf{j}, s}+\text { H.c. }\right)+J \sum_{\langle\mathbf{i}, \mathbf{j}\rangle}\left(\mathbf{S}_{\mathbf{i}} \mathbf{S}_{\mathbf{j}}-\frac{1}{4} n_{\mathbf{i}} n_{\mathbf{j}}\right) \\
& +g \sum_{\mathbf{i}, \boldsymbol{\delta}}\left(n_{\mathbf{i}}^{h}-n_{\mathbf{i}+\boldsymbol{\delta}}^{h}\right)\left(a_{\mathbf{i}+\boldsymbol{\delta} / 2}^{\dagger}+a_{\mathbf{i}+\boldsymbol{\delta} / 2}\right) \\
& +q_{\beta} \sum_{\mathbf{i}, \boldsymbol{\delta}}\left(n_{\mathbf{i}}^{h}+n_{\mathbf{i}+\boldsymbol{\delta}}^{h}\right)\left(a_{\mathbf{i}+\boldsymbol{\delta} / 2}^{\dagger}+a_{\mathbf{i}+\boldsymbol{\delta} / 2}\right)^{\beta} \\
& +\omega_{0} \sum_{\mathbf{i}+\boldsymbol{\delta}} a_{\mathbf{i}+\boldsymbol{\delta} / 2}^{\dagger} a_{\mathbf{i}+\boldsymbol{\delta} / 2}
\end{aligned}
$$

where $\tilde{c}_{\mathbf{i}, s}=c_{\mathbf{i}, s}\left(1-n_{\mathbf{i},-s}\right)$ is a projected fermion operator, $t$ represents nearest neighbor overlap integral, the 
$\operatorname{sum}\langle\mathbf{i}, \mathbf{j}\rangle$ runs over pairs of nearest neighbors, $a_{\mathbf{i}}$ are phonon annihilation operators and $n_{\mathbf{i}}=\sum_{s} n_{\mathbf{i}, s}$. The third term represents linear EP coupling to LP of O vibration with respect to $\mathrm{Cu}-\mathrm{O}-\mathrm{Cu}$ bond, see also Fig. 1(a). Fourth term is chosen either linear $(\beta=1)$ or quadratic $(\beta=2)$ in $\mathrm{O}$ displacement, describing TP of $\mathrm{O}$ vibration, Fig. 1(a). $\beta=2$ is chosen to describe the $\mathrm{CuO}$ plane with no pre-buckling of O positions. Sums over $\boldsymbol{\delta}$ in the latter two terms run over two orthogonal nearest neighbor $\mathrm{Cu}$ positions. Lattice vibrations on $\mathrm{O}$ sites are independent - we do not predispose any particular phonon mode with the exception of limiting our calculation to either TP or $\mathrm{LP}$ of $\mathrm{O}$ oscillation. In treating quantum phonons we follow well established approach of Ref. [30].

The construction of the functional space starts from a Néel state with two holes located on neighboring $\mathrm{Cu}$ sites and with zero phonon quanta. Such a state represents a parent state of a translationally invariant state with a given momentum $k$. In the case of a high symmetry point $\mathbf{k}=(0,0)$ the parent state can be chosen to have $d-, s-$, or $p-$ wave symmetry as for the case of $d-$ and $s-$ shown in Fig. 1(b). The starting state is written as $\left|\phi^{(0)}\right\rangle_{a}=\sum_{\gamma}(-1)^{M_{a}(\gamma)} c_{0} c_{\gamma} \mid$ Neel; 0$\rangle$, where sum runs over four nearest neighbors in the case of $d$ - and $s$ - wave symmetry and over two in the case of $p_{x(y)}$-wave while $M_{a}(\gamma), a \in\{d, s, p\}$ sets the appropriate sign.

We generate new parent states by applying the generator of states $\left\{\left|\phi_{l}^{\left(n_{h}\right)}\right\rangle_{a}\right\}=$ $\left(H_{\mathrm{kin}}+\tilde{H}_{J}+H_{\mathrm{ph}}\right)^{n_{h}}\left|\phi^{(0)}\right\rangle_{a} ; n_{h}=1, \ldots, N_{h}$ where $H_{\text {kin }}$ represent the first term in Eq. 1, $\tilde{H}_{J}$ denotes a part of the second term in Eq. 1 which is only applied to erase spin flips that were generated through succeeding application of $H_{\text {kin }}$, as for a particular case depicted in Fig. 1(c). $H_{\mathrm{ph}}$ represents either third or fourth term in Eq. 1. This procedure generates exponentially growing basis of states, consisting of different shapes of strings in the vicinity of the hole with maximum lengths given by $N_{h}$ as well as phonon quanta created along paths of both holes. Identical basis functions, generated by different processes, are chosen only once. We have used $N_{h}=8$ that lead to $N_{\text {st }}=13 \times 10^{6}$ states. Full Hamiltonian in Eq. 11 is diagonalized within this limited functional space taking explicitly into account translational symmetry.

In Fig. 2 we present the energy difference $E_{p}-E_{d}$ between the lowest $p$ - and the $d$-wave state for two different values of $J / t$ as a function of $q_{\beta} / t$ and $g / t$ for the case of TP and LP respectively. At $J / t=0.1$ the two-holes are unbound at $q_{1,2}=g=0[22,23]$ and degenerate $p$-wave ground state is found, $E_{p}-E_{d}<0$, see Figs. 2(a) and (b). Increasing $q_{\beta} / t$ and $g / t$ leads to rather surprisingly distinct results. In both cases increasing EP coupling leads to a formation of a bipolaron, as also evident from Figs. 3(e) and (f) and the discussion later in the text. While coupling to TP leads to a

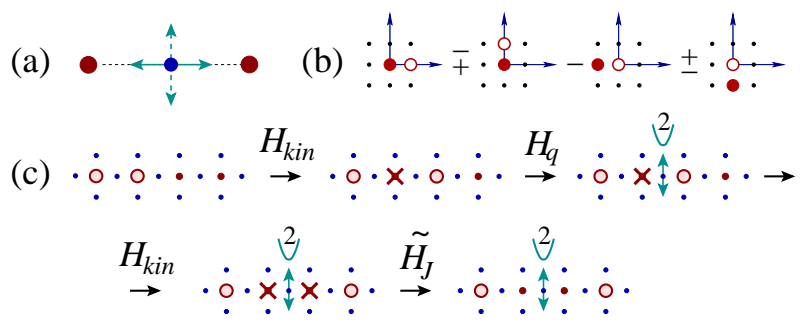

Figure 1: (Color online) (a) Schematic representation of LP and $\mathrm{TP}$ vibrations of $\mathrm{O}$ atom (middle) with respect to $\mathrm{Cu}-$ $\mathrm{O}-\mathrm{Cu}$ bond, (b) schematic representation of a $d_{x^{2}-y^{2}}$-wave ( $s$-wave) top (bottom) signs two-hole starting wavefunction. Fermion sign convention places the first hole depicted with the full circle to the left-most, if the pair is vertical, then bottommost position. Only $\mathrm{Cu}$ sites are presented with small dots in (b); (c) schematic representation of succeeding applications of different off-diagonal parts of Hamiltonian in Eq. 1. for $n_{h}=4$, starting from a single hole-pair in the Néel state with zero phonon quanta. Dots represent $\mathrm{Cu}$ and $\mathrm{O}$ atoms, holes are denoted by open circles, crosses represent spin-flips (overturned spins with respect to the original Néel configuration of spins, localized on $\mathrm{Cu}$ sites), vertical arrows indicate and point to the numbers of excited TP phonon quanta.

formation of a bound state with the $d$-wave symmetry, coupling to LP in contrast favors a bound state with the $p$-wave symmetry. This effect is even more pronounced at larger value of $J / t=0.4$ where at $q_{1,2}=g=0$ a bound magnetic bipolaron is already formed [22, 23, 24] with a $d$-wave symmetry. By increasing $q_{2} / t, d$-wave symmetry is stabilized. Increasing linear EP coupling $q_{1} / t$ leads to an initial increase of $E_{p}-E_{d}$ followed by a decrease, $E_{p}-E_{d} \rightarrow 0$ around $q_{1} / t \gtrsim 0.75$ due to a crossover to a strong EP coupling regime, Fig. 2(a). In contrast, linear EP coupling to LP drives even a bound $d$-wave bipolaron state at $J / t=0.4$ and $g=0$ to a bound state with a $p$-wave symmetry at $g / t \sim 0.61$, see Fig. 2(b).

Effective bipolaron mass $m_{\alpha \alpha}=t\left[\partial^{2} E(\mathbf{k}) / \partial \mathbf{k} \partial \mathbf{k}\right]_{\alpha \alpha}^{-1}$, computed in its eigen-directions, presented in Figs. 2(c) and (d), is isotropic in the case of $d$-wave symmetry and anisotropic, with the anisotropy ratio $m_{y y} / m_{x x} \sim 3-10$ in the case of $p$-wave state. At $J / t=0.4 m_{\alpha \alpha}$ furthermore shows only a weak increase with $q_{2} / t$, see Fig. 2(c). Even more surprising is the decrease of the effective mass at $J / t=0.1$ in the regime of a bound $d$-wave bipolaron, i.e. for $q_{2} / t \gtrsim 0.5$. Note, that the nonanalytic behavior of $m_{x x}$ is a consequence of the symmetry change from $p-$ to $d-$ state at $q_{2} / t \sim 0.5$ as also seen from Fig. 2 (a).

Focusing on linear EP coupling to LP, $m_{x x}$ at $J / t=0.4$ starts a rapid increase signaling the approach to strong EP coupling regime just below the transition to the $p$-wave state, around $g / t \sim 0.57$, see Fig. 2(d). As the system enters $p$-wave state the mass again becomes anisotropic.

In Fig. 3 we present the probability of finding a hole-pair at a distance of $\mathrm{r}: P(r)=$ 


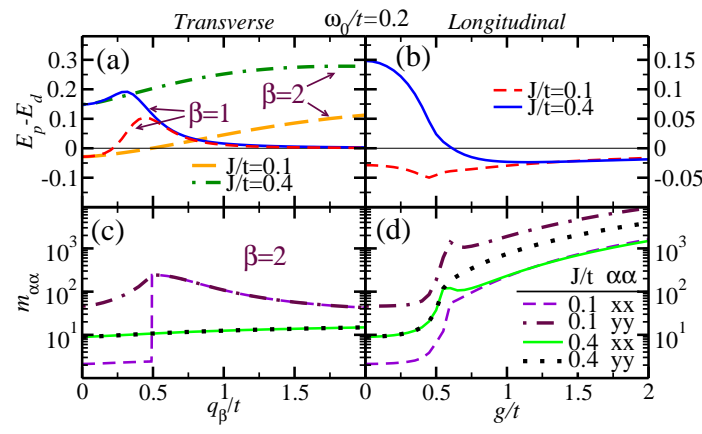

Figure 2: (Color online) $E_{p}-E_{d}$ at $\omega_{0} / t=0.2$, and two different strengths of $J / t$ vs. $q_{\beta} / t$ in the case of EP coupling to TP (a) and vs. $g / t$ in the case of linear EP coupling to LP (b). Effective masses $m_{\alpha \alpha}$ vs. $q_{2} / t$ in (c) and vs. $g / t$ in (d). The ground state wave-vector is $\mathbf{k}=0$ except in the regime $g / t \gtrsim 0.57$ where $\mathbf{k}=(\pi, 0)$.

$\left\langle\sum_{\langle\mathbf{i} \neq \mathbf{j}\rangle} n_{\mathbf{i}}^{h} n_{\mathbf{j}}^{h} \delta[|\mathbf{i}-\mathbf{j}|-r]\right\rangle /\left\langle\sum_{\langle\mathbf{i} \neq \mathbf{j}\rangle} n_{\mathbf{i}}^{h} n_{\mathbf{j}}^{h}\right\rangle$, and average hole distance $\langle d\rangle=\sum_{r} r P(r)$. We first focus on the effect of EP coupling to TP, see Figs. 3(a,c,e). At $J / t=0.1$, bipolaron is unbound in the regime $\left(q_{2} / t \lesssim 0.5\right.$, and $\left.q_{1} / t \lesssim 0.22\right)$, nevertheless, $\langle d\rangle$ remains finite due to a limited Hilbert space where the maximal inter-hole distance is given by $l_{\max }=N_{h}+1=9$. Increasing $N_{h}$ would lead to further increase of $\langle d\rangle$ in this regime as well as to further spread of $P(r)$ towards larger $r$, see Fig. 3(c) for $J / t=0.1$ and $q_{1,2}=0$. In this range of parameters we observe no exponential decay of $P(r)$, see Fig. 3(e). In contrast, in the regime of a bound bipolaron, i.e. for $J / t=0.1$ and $\left(q_{2} / t \gtrsim 0.5\right.$ and $\left.q_{1} / t \gtrsim 0.22\right)$ as well as at $J / t=0.4,\langle d\rangle$ and $P(r)$ do not change much with further increasing $N_{h}$ and exponential decay is clearly observed in Fig. 3(e). Our tests performed on smaller systems $\left(N_{h}=4\right.$ and 6$)$ reaffirm that results in the regime of a bound bipolaron have indeed converged close to a thermodynamic limit. Good agreement of $P(r)$ at $J / t=0.4$ and $q_{1,2}=g=0$ is found with ED calculation on 32-sites cluster, Ref. [22, 24]. Structure of a bound bipolaron, revealed by $P(r)$ at $J / t=0.4$ and $q_{1,2}=0$, is remarkably similar to that computed at $J / t=0.1$ and $q_{2} / t=1.0$ and $\beta=2$, Fig. B(c). Both, quadratic and linear EP coupling to TP lead to a formation of a bound bipolaron with the $d$-wave symmetry even in the case of small exchange interaction $J / t=0.1$ where magnetic mechanism is not strong enough to form a bound magnetic bipolaron. To investigate whether coupling to TP alone can lead to $d$-wave state in the absence of a magnetic background, we have solved a problem with two spinless particles quadratically coupled to TP or linearly to LP using topology of a $\mathrm{Cu}-\mathrm{O}$ plane. By increasing $q_{2} / t$ or $g / t$ we obtain in both cases a bipolaron with a $p$-wave symmetry, Figs. 3(a) and (b). We thus emphasize an important conclusion: EP coupling to TP stabilizes $d$-wave symmetry of a hole-pair, however, the existence of a mag- netic background as found in the $t-J$ model seems to be essential precondition for the formation of a $d$-wave state.
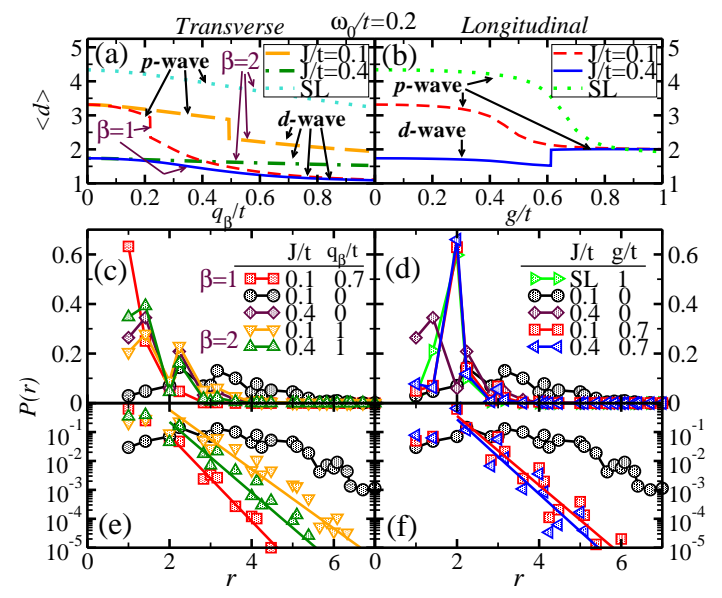

Figure 3: (Color online) Average hole distance $\langle d\rangle$ computed at $\omega_{0} / t=0.2$ and two different values of $J / t$ vs. $\quad q_{\beta} / t ; \beta=1,2$ in (a) and vs. $g / t$ in (b). Results for the system of spinless particles (SL) are shown with dotted lines in (a) and (b); $P(r)$ at chosen values of $q_{1,2} / t$ in (c) and $g / t$ in (d). $P(r)$ is normalized to $\sum_{r} P(r)=1$; corresponding exponential scalings of $P(r)$ for $r \gtrsim 2$ are shown in (e) and (f).

Turning to linear EP coupling to LP phonons we start from $J / t=0.1$. A $p$-state of two separate holes changes to a bound bipolaron state with increasing of $g / t$ at about $g / t \sim 0.5$, see Fig. 3(b). Transition to a bound state is not sharp as in Fig. 3(a) since there is no change of a symmetry. Nevertheless, in the $N_{h} \rightarrow \infty$ limit, we anticipate a sharp transition from an unbound to a bound bipolaron state. Stabilization of a $p$-wave state under the influence of LP is even more evident when starting from a $d$-wave bound bipolaron state at $J / t=0.4$. With increasing $g / t$, a change of symmetry occurs around $g / t \sim 0.61$, from a $d$ - to a $p$-wave state, see Figs. [3(b,d,f). A detailed inspection of a bound $p$-wave state in the regime $g / t \gtrsim 0.61$ reveals unusually simple structure where the probability of finding holes at a distance $r=2$ is more than 0.6. This is in a sharp contrast with the structure of a $d$-wave bound state where $P(r=2)<0.1$ and the maximal value of $P(r)$ is at $r=\sqrt{2}$, compare also Figs. 4(a) and (b). Our calculations of hopping term modulated by LP phonons, as suggested in Ref. [13] , as well leads to stabilization of a bipolaron with a $p$-wave symmetry, nonetheless, with a distinct spacial structure.

From Fig. 3 is as well evident that linear coupling to TP leads a stronger attraction between holes than coupling to LP as seen from Figs. 3(e) and (f) that show steeper decay of $P(r)$ for TP at comparable values $q_{1} / t=g / t=0.7$. At small $J / t=0.1$ a bound bipolaron state is obtained at unexpectedly small value of the dimensionless EP coupling constant $\lambda_{q}=q_{1}^{2} / 4 \omega_{0} t \sim 0.06$ in the TP case in contrast to $\lambda_{g}=g^{2} / 4 \omega_{0} t \sim 0.31$ in the 
LP case. This result suggests that even a small prebuckling within the $\mathrm{CuO}$ plane, that generates non-zero linear EP coupling term to TP, may have a pronounced effect on the attraction between magnetic polarons.

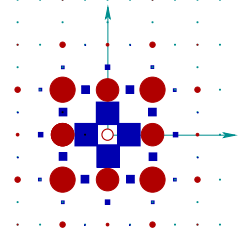

(a)

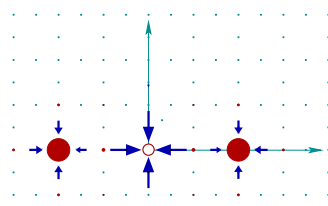

(b)
Figure 4: (Color online) (a) $\mathcal{C}(\mathbf{r})$ and $\mathcal{N}(\mathbf{r})$ at $J / t=0.4$, $q_{2} / t=1.0$, and $\beta=2$ ( $d$-wave). Radii of circles, representing $\mathcal{C}(\mathbf{r})$, located on $\mathrm{Cu}$-sites are, proportional to the probability of finding a hole-pair at a distance of $\mathbf{r}$. Empty circle is located at $\mathbf{r}=0$. Sides of squares, representing $\mathcal{N}(\mathbf{r})$, located on $\mathrm{O}$ sites, are proportional to average numbers of phonon quanta at a distance $\mathbf{r}$ from a hole; (b) $\mathcal{C}(\mathbf{r})$ and $\mathcal{X}(\mathbf{r})$ at $J / t=0.4$ and $g / t=0.7$ ( $p$-wave). Lengths of arrows, representing $\mathcal{X}(\mathbf{r})$, are proportional to displacements of $\mathrm{O}$ atoms along $\mathrm{Cu}-\mathrm{Cu}$ bonds relative to the hole position.

To investigate in more detail the nature of the magnetic-lattice bipolaron, we simultaneously present two correlation functions: hole-hole density $\mathcal{C}(\mathbf{r})=\left\langle\sum_{\mathbf{i}} n_{\mathbf{i}}^{h} n_{\mathbf{i}+\mathbf{r}}^{h}\right\rangle$, and hole-phonon number $\mathcal{N}(\mathbf{r})=$

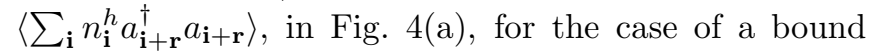
$d$-wave bipolaron state. Largest phonon numbers are found at the closest possible distance from the hole. The structure of $\mathcal{C}(\mathbf{r})$ is consistent with $d_{x^{2}-y^{2}}$ symmetry despite its largest value at a distance of $r=\sqrt{2}$, as already pointed out in Refs. 23, 24]. In Fig. 四(b) we show $\mathcal{C}(\mathbf{r})$ and $\mathcal{X}(\mathbf{r})=\left\langle\sum_{\mathbf{i}} n_{\mathbf{i}}^{h}\left(a_{\mathbf{i}+\mathbf{r}}^{\dagger}+a_{\mathbf{i}+\mathbf{r}}\right)\right\rangle$, measuring displacements along $\mathrm{Cu}-\mathrm{Cu}$ bonds relative to the position of the hole, for the case of a $p$-wave ground state. Both correlations display the unidirectional spacial distribution. Correlation functions, presented in Figs. 4(a) and (b), show detectable values only up to $r \lesssim 3$, despite maximal distance $l_{\max }=N_{h}+1=9$, allowed in our calculations.

In conclusion significantly different bipolaron states are found when EP coupling to either TP or LP is switched on. Linear as well as quadratic EP coupling to TP stabilizes a $d$-wave bipolaron state. The magnetic background is essential for the formation of a $d$-wave bipolaron. The effective bipolaron mass remains small in the case of quadratic EP coupling despite lattice driven binding of the bipolaron.

In contrast, increasing linear EP coupling to LP phonons changes the symmetry of a bound bipolaron from a $d$-wave state at zero EP coupling to a $p$-wave state followed by a substantial change of the densitydensity correlation function. Since this state also has a large and anisotropic effective mass and unidirectional spacial distribution we may speculate, that in a system with finite doping linear EP coupling to LP of O vibration would lead to formation of stripe states. This finding is consistent with inelastic neutron experiments showing strong coupling to the bond-streching mode in and around the vicinity of the stripe phase in copper oxide superconductors [5].

J.B. acknowledges financial support of the SRA under grant P1-0044. S.M. and T.T. acknowledge the financial support of the Next Generation Super Computing Project of Nanoscience Program, CREST, and Grant-inAid for Scientific Research from MEXT. This work was also supported by JPSJ and MHEST under the JapanSlovenia Research Cooperative Program.

[1] A. Lanzara et al., Nature 412, 510 (2001).

[2] A. Alexandrov and N. F. Mott, Rep. Prog. Phys. 57, 1197 (1994).

[3] D. M. Newns and C. C. Tsuei, Nature Physics 3, 184 (2007).

[4] O. Gunnarsson and O. Rosch, Journal of Physics: Condensed Matter 20, 043201 (22pp) (2008).

[5] D. Reznik et al., Nature 440, 1170 (2006).

[6] F. Ronning et al., Phys. Rev. B 71, 094518 (2005).

[7] J. Bonča et al., Phys. Rev. B 77, 054519 (2008).

[8] A. S. Alexandrov, Phys. Rev. B 77, 094502 (2008).

[9] J. Riera and A. Moreo, Phys. Rev. B 73, 014518 (2006).

[10] T. Sakai, D. Poilblanc, and D. J. Scalapino, Phys. Rev. B 55, 8445 (1997).

[11] P. Prelovšek, R. Zeyher, and P. Horsch, Phys. Rev. Lett. 96, 086402 (2006).

[12] M. Hohenadler et al., Phys. Rev. B 71, 245111 (2005).

[13] S. Ishihara and N. Nagaosa, Physi. Rev. B 69, 144520 (2004).

[14] B. Kyung et al., Phys. Rev. B 54, 13167 (1996).

[15] A. Ramšak, P. Horsch, and P. Fulde, Phys. Rev. B 46, 14305 (1992).

[16] G. Sangiovanni et al., Phys. Rev. Lett. 97, 046404 (2006).

[17] A. Macridin et al., Phys. Rev. Lett. 97, 056402 (2006).

[18] E. Cappelluti, S. Ciuchi, and S. Fratini, Phys. Rev. B 76, 125111 (2007).

[19] G. D. Filippis et al., Phys. Rev. Lett. 99, 146405 (2007).

[20] A. S. Mishchenko and N. Nagaosa, Phys. Rev. Lett. 93, 036402 (2004).

[21] O. Rosch and O. Gunnarsson, Phys. Rev. Lett. 92, 146403 (2004).

[22] A. L. Chernyshev, P. W. Leung, and R. J. Gooding, Phys. Rev. B 58, 13594 (1998).

[23] P. Wróbel and R. Eder, Phys. Rev. B 58, 15160 (1998).

[24] J. Riera and E. Dagotto, Phys. Rev. B 57, 8609 (1998).

[25] H. Barentzen and V. Oudovenko, Europhys. Lett. 47, 227 (1999).

[26] $d$-wave symmetry of a bipolaron is a many-body effect. A bound state of two particles with attractive interaction is always nodeless ( $s$-wave in $2 \mathrm{D}$ ), see also Ref. [8].

[27] P. W. Leung, Phys. Rev. B 65, 205101 (2002).

[28] T. Aimi and M. Imada, J. Phys. Soc. Jpn. 76, 113708 (2007).

[29] J. Bonča, S. Maekawa, and T. Tohyama, Phys. Rev. B 76, 035121 (2007).

[30] J. Bonča, S. A. Trugman, and I. Batistić, Phys. Rev. B 
60, 1633 (1999). 